\title{
Numerical investigation on the induction zone structure of the oblique detonation waves
}

\author{
Honghui Teng ${ }^{\mathrm{a}, *}$, Yining Zhang ${ }^{\mathrm{b}}$, Zonglin Jiang ${ }^{\mathrm{a}}$ \\ a State Key Laboratory of High Temperature Gas Dynamics, Institute of Mechanics, Chinese Academy of Sciences, Beijing 100190, China \\ ${ }^{\mathrm{b}}$ State Key Laboratory of Laser Propulsion \& Application, Beijing Power Machinery Research Institute, Beijing 100074, China
}

\section{A R T I C L E I N F O}

\section{Article history:}

Received 29 July 2013

Received in revised form 4 February 2014

Accepted 1 March 2014

Available online 11 March 2014

\section{Keywords:}

Detonation

Oblique shock

Induction

Unstationary

\begin{abstract}
A B S T R A C T
Oblique detonation waves are simulated to study the induction zone structures with different incident Ma numbers. Three kinds of shock configurations are observed at the end of the induction zone, which are the $\lambda$-shaped shock, the X-shaped shock and the Y-shaped shock. The X-shaped and Y-shaped shocks appear when the incident $M a$ is low, and the Y-shaped shock associated with the complicated unstationary process. The induction zone length reaches the maximum value when the $\mathrm{X}$-shaped shock changes into the Y-shaped shock, which indicates different mechanisms deciding the induction zone. The oblique shock wave dominates the induction zone when the incident $M a$ is high, while the oblique detonation wave dominates the induction zone when the incident $M a$ is low.
\end{abstract}

๑) 2014 Elsevier Ltd. All rights reserved.

\section{Introduction}

High efficient propulsion systems are key technologies in the design of air-breathing hypersonic aircrafts. One of the propulsion concepts is based on the oblique detonation wave, which derives into the Oblique Detonation Wave Engines (ODWE) and the Ram Accelerators [1]. This kind of propulsion system not only has the advantages of the Scramjet (Supersonic combustion ramjet), but also achieves high thermal cycle efficiency through the detonation $[2,3]$. Therefore the oblique detonation propulsion attracts much attention for its potential application in the hypersonic. In the early research, oblique detonation waves are usually simplified to be the oblique shock waves and post shock release zones attached on the wedge [4]. However, this assumption ignores the realistic structure near the wedge front tip, which may be problematic. Li et al. [5] studied the oblique detonation numerically, and observed the structure composed of a nonreactive oblique shock, an induction region, a set of deflagration waves, and the oblique detonation surface. This structure is verified experimentally [6] and then considered to be the standard structure used widely in recent years. Papalexandris et al. [7] have found out that the triple points may appear on the detonation surface in the high activation energy gas. Choi et al. [8] studied these small scale structures and demonstrated the coupling relation between the shock and the combustion. Teng et al. [9] found out the formation of triple points

\footnotetext{
* Corresponding author. Tel.: +86 10 82544011; fax: +86 1082543995 .

E-mail address: honghuiteng@gmail.com (H. Teng).
}

is hard in the case of high overdrive degree, but it derives from small disturbance and cannot be suppressed thoroughly. The other kind of small scale structures, which is similar to the detonation cell on the normal detonation surface, are also observed and discussed recently [10-12].

Although the oblique detonation surface has been studied widely, the oblique shock-detonation transition is not understood deeply. This transition can be viewed as the oblique detonation initiation, so it is the origin of the complicated surface of the oblique detonation. Generally there are two kinds of transition structures, the abrupt one and the smooth one. The transition structures have been studied and the criterions on the transition type have been proposed [13-15]. However, most of previous researchers studied on the static structures, which are assured by the high incident Mach numbers (Ma). The more complicated structures, such as the divergent $\lambda$-shaped shock, would be induced by the transition in the case of a low Ma [16]. Nevertheless, only the static structure is given before and the evolution of this structure when $M a$ decreases further is still unclear. These structures are probably coupled with the unstationary process when incident Ma decreases, and their evolutions are very important in the oblique detonation propulsion design. In this paper, the structure evolutions are simulated and their relationships are discussed to ascertain the structure characteristics of the induction zone.

\section{Mathematical and physical models}

Sketch of oblique detonation wave induced by the wedge in the combustible gas mixtures is shown in Fig. 1. Supersonic 


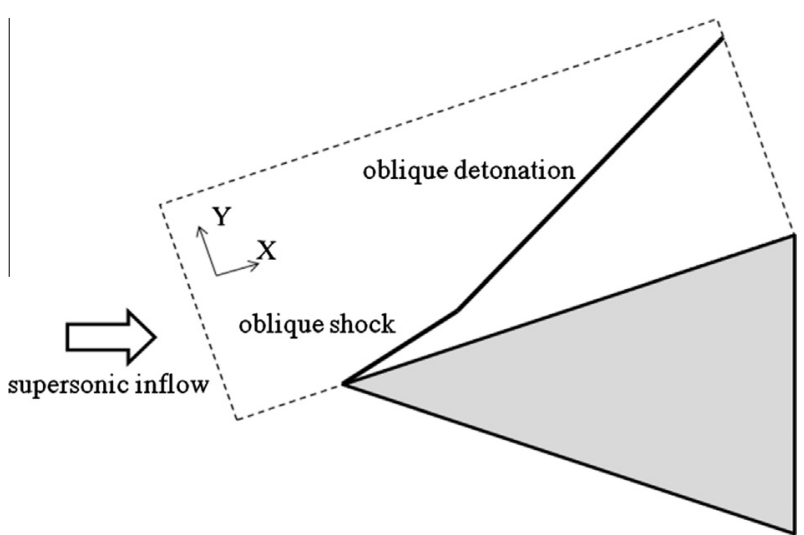

Fig. 1. Schematic of wedge-induced oblique detonations in the combustible gas mixtures.

combustible gas mixtures reflect on the two-dimensional wedge and generate an oblique shock wave first. The shock wave may induce the exothermic chemical reaction, and then a complicated detonation structure will form downstream. The computational simulation is carried out in the dashed zone shown in Fig. 1, whose coordinate is rotated to the direction along the wedge surface. Previous results $[13,17]$ showed that the viscosity and boundary layer have little effects on this structure except changing the boundary layer thickness slightly, and most of the results use the inviscid calculation. Then the governing equations are simplified as two-dimensional multi-species Euler equations and can be written as follows:

$\frac{\partial \mathbf{U}}{\partial t}+\frac{\partial \mathbf{F}}{\partial x}+\frac{\partial \mathbf{G}}{\partial y}=\mathbf{S}$

where

$\mathbf{U}=\left\{\begin{array}{c}\rho_{1} \\ \vdots \\ \rho_{n} \\ \rho u \\ \rho v \\ e\end{array}\right\}, \mathbf{F}=\left\{\begin{array}{c}\rho_{1} u \\ \vdots \\ \rho_{n} u \\ \rho u^{2}+p \\ \rho u v \\ (e+p) u\end{array}\right\}, \mathbf{G}=\left\{\begin{array}{c}\rho_{1} v \\ \vdots \\ \rho_{n} v \\ \rho u v \\ \rho v^{2}+p \\ (e+p) v\end{array}\right\}, \mathbf{S}=\left\{\begin{array}{c}\omega_{1} \\ \vdots \\ \omega_{n} \\ 0 \\ 0 \\ 0\end{array}\right\}$

In above equations $\rho_{i}(i=1, \ldots, n)$ is the $i$ th specie density and the total density $\rho=\sum_{i=1}^{n} \rho_{i} ; u$ and $v$ are the velocity in the $x$ - and $y$-direction. Total specific energy $e$ is calculated by

$e=\rho h-p+\frac{1}{2} \rho\left(u^{2}+v^{2}\right)$

where specific enthalpy can be written as $h=\sum_{i=1}^{n} \rho_{i} h_{i} / \rho$ and the $i$ th specie specific enthalpy $h_{i}$ can be got by curve fitting; $p$ stands for gas pressure and equation of state is

$p=\sum_{i=1}^{n} \rho_{i} R_{i} T$

where $R_{i}$ is the $i$ th specie gas constant and $T$ is the gas temperature; $\dot{\omega}_{i}$ is the $i$ th specie specific mass production rate, which is decided by the chemical reaction model.

Governing equations are solved on adaptive unstructured quadrilateral grids [18] with MUSCL-Hancock scheme [19]. This scheme achieves the second-order accurate in space and time by constructing the Riemann problem on the intercell boundary through the equations

$$
\begin{aligned}
& \bar{U}_{i}^{L}=U_{i}^{L}+\frac{\Delta t}{2 \Delta x}\left[F\left(U_{i}^{L}\right)-F\left(U_{i}^{R}\right)\right] \\
& \bar{U}_{i}^{R}=U_{i}^{R}+\frac{\Delta t}{2 \Delta x}\left[F\left(U_{i}^{L}\right)-F\left(U_{i}^{R}\right)\right]
\end{aligned}
$$

And the solution is computed by HLLC approximate Riemann solver. The computational domain is $2.0 \mathrm{~cm} \times 0.5 \mathrm{~cm}$, based on the coarsest mesh $1.0 \mathrm{~mm} \times 1.0 \mathrm{~mm}$. 5-level refinement adopted by adaptive mesh refinement (AMR) method, which induces the finest grid about $0.031 \mathrm{~mm}$.

Hydrogen/air chemical reaction model [20] is selected from the widely used CHEMKIN package and 11 species $\left(\mathrm{H}_{2}, \mathrm{O}_{2}, \mathrm{O}, \mathrm{H}, \mathrm{OH}\right.$, $\mathrm{HO}_{2}, \mathrm{H}_{2} \mathrm{O}_{2}, \mathrm{H}_{2} \mathrm{O}, \mathrm{N}_{2}, \mathrm{~N}, \mathrm{NO}$ ) and 23 reactions are account for in chemical reactions. The stiff problem due to chemical reaction calculation is solved by the DVODE package [21]. The slip reflecting boundary condition is used on the wedge surface and the other boundaries are interpolated under the assumption of the zero first-order derivatives of all flow parameters. The wedge starts from $x=0.5$ and the length scale is $\mathrm{cm}$ in the figures shown later. The initial gas is hydrogen/air premixed mixtures with $\mathrm{H}_{2}: \mathrm{O}_{2}: \mathrm{N}_{2}=2: 1: 4$, temperature $298.15 \mathrm{~K}$, and pressure $101325 \mathrm{~Pa}$. Gas velocities in the $x$ - and $y$-direction are decided by incident Mach numbers and wedge angle, which is fixed to be $30^{\circ}$ in this simulation. Parameters aforementioned are implemented on the whole flow field initially.

\section{Numerical results and discussion}

\subsection{Initiation and structures with Ma 7.0}

The initiation process of oblique detonation wave structure with the incident Ma 7.0 is shown in Fig. 2. With the uniformed inflow, the reaction starts around $x>1.0$ and reaction front propagates upstream in the shocked flow, as shown in Fig. 2a. After oblique shock forms, the reaction front moves downstream, and a normal detonation can be observed in shocked flow, as show in Fig. $2 \mathrm{~b}$. The detonation splits and forms the structure characterized by the $\lambda$-shaped shock at the end of the induction zone, as shown in Fig. $2 c$ and d. This structure is also shown in the previous letter [16]. Fig. 3 shows the shock and reaction zone indicated by pressure and $\mathrm{OH}$ radical density, respectively. It can be found out the transition from the oblique shock to the oblique detonation wave is achieved by one multi-wave point, which is associated with the divergent shock wave below. One short Mach stem can be observed derives from the multi-point above two shock branches. The upstream branch of the divergent shock couples with the deflagration wave, and the other reflects in the combustion product.

Previous researchers use the grid number in half reaction length to evaluate the resolution of detonation simulations, and in recent studies the resolution of 32 grids half reaction length is usually used [22,23]. Unfortunately, we use the detailed reaction mechanism, which is hard to give the half reaction length because there is no "reaction progress" in these mechanisms. The reactions are reversible, and even the heat release is implicit in the mechanisms and dependent on the final states. To evaluate the resolution, we examined the density of intermediate radical $\mathrm{OH}$, which rises behind the shock and then decreases in the process of heat release. In the case of $M a 7.0$, it takes about $0.25 \mathrm{~mm}$ to reach its maximum value behind the shock wave. Therefore, there are about 8 grids in half reaction length in this study.

It should be noticed the resolution used in oblique detonations and 2D normal detonations are usually different. As mentioned above, 32 grids are usually used in recent 2D normal detonations, but much less grids used in recent oblique detonations. For example, Choi et al. [8] use about 5 grids in half reaction length in the unstable surface studies, and Verreault et al. [12] use the 

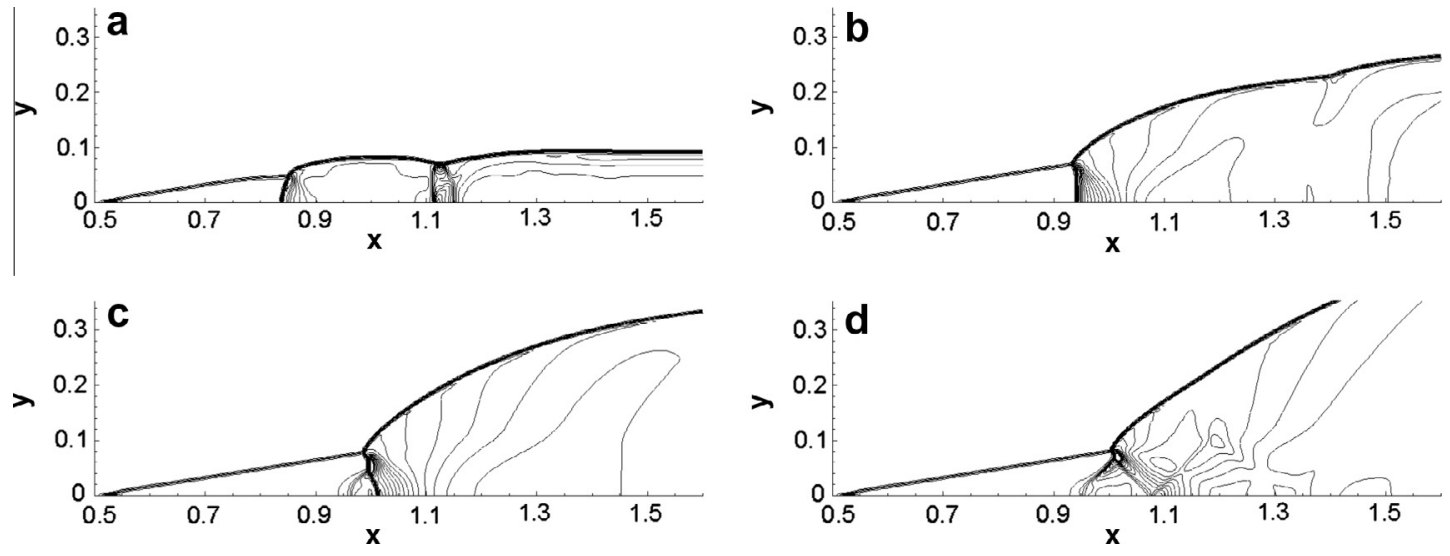

Fig. 2. Pressure of the oblique detonation wave formation with the incident Ma 7.0.

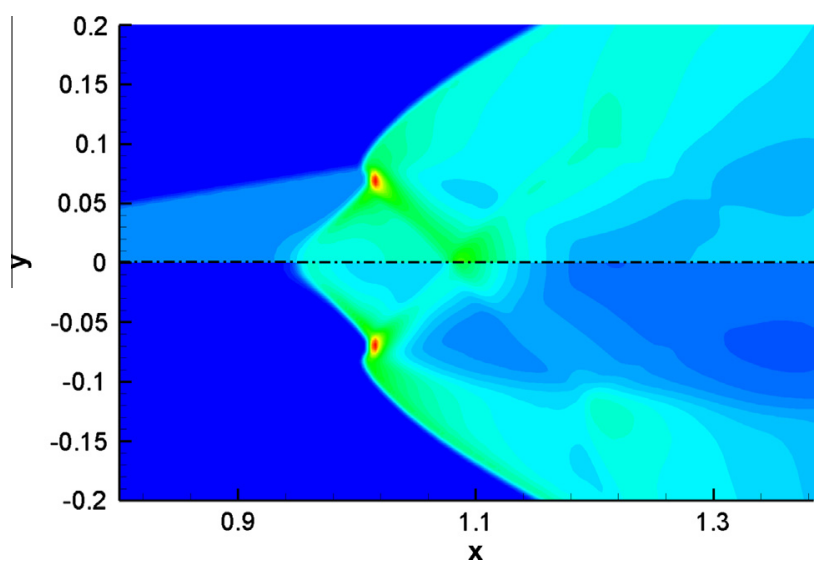

Fig. 3. Pressure (upper) and $\mathrm{OH}$ density (lower) of the oblique detonation wave with the incident $M a 7.0$.

finest 13 grids to study the formation of transverse waves on the oblique detonation surface. Hence, it seems oblique detonation needs less resolution in the numerical simulation, although the reason is still not clear now. Moreover, the induction zone structure studied here is different from small-scale waves on the oblique detonation surface studied before. Theoretically this study needs less resolution because the instability is not triggered in our computational domain. Therefore, the resolution with about 8 grids in half reaction length is enough to capture the induction zone structure, which will be used in the later part of this paper.

\subsection{Effects of incident $M a$}

The oblique detonation wave with the incident $M a 6.8$ is shown in Fig. 4. Compared with last case, the transition position from the oblique shock to detonation wave moves downstream and the induction zone length rises from about $0.5 \mathrm{~cm}$ to about $1.0 \mathrm{~cm}$. Furthermore, the change of the shock wave configuration is also observed. The Mach stem becomes longer than the last one, and the new branch forms to induce the X-shaped shock configuration, which has never been observed before. Decreasing the Ma further to 6.7 , another kind of shock configuration can be observed, as shown in Fig. 5. The new branch grows longer although the induction zone length changes little. Original divergent branches couple together, which induces the structure characterized by the Y-shaped shock. These three kinds of shock configurations, which are $\lambda$-shaped, $\mathrm{X}$-shaped, and $\mathrm{Y}$-shaped, demonstrate the diversity of oblique detonations with low Mach number.

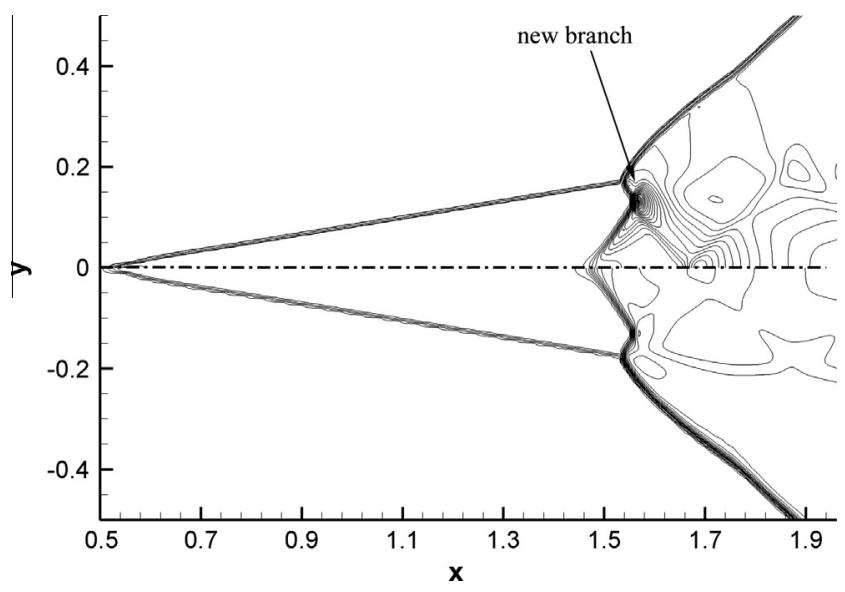

Fig. 4. Pressure (upper) and temperature (lower) of the oblique detonation wave with the incident $M a 6.8$.

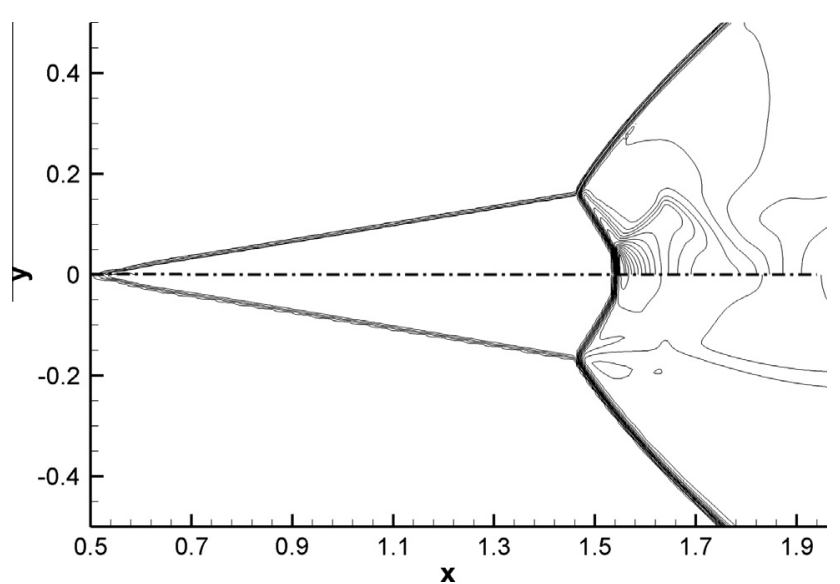

Fig. 5. Pressure (upper) and temperature (lower) of the oblique detonation wave with the incident $M a 6.7$.

Because the X-shaped shock configuration can be viewed as a transition pattern, the difference between the $\lambda$-shaped one and the Y-shaped one should be analyzed. In the $\lambda$-shaped case, there is only weak shock or compression wave around the reaction front wave near the wedge, see Figs. 2 and 3. The ignition there is mainly achieved by the oblique shock compression, i.e. auto-ignition. When the incident $\mathrm{Ma}$ decreases, corresponding auto-ignition 


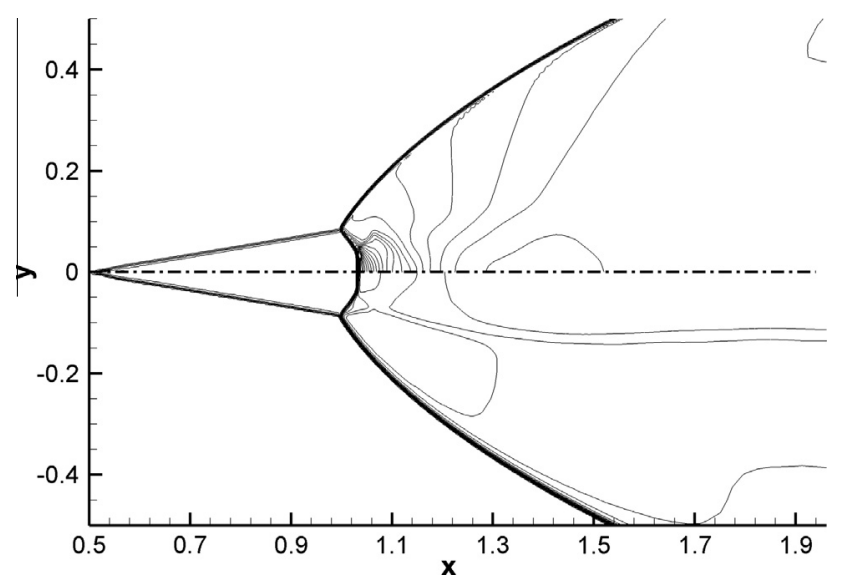

Fig. 6. Pressure (upper) and temperature (lower) of the oblique detonation wave with the incident $M a$ 6.6.

position moves downstream due to its sensitivity to the temperature. However, low Ma induces the large oblique detonation angle, which intends to move the position upstream. The competition is compromised by a normal detonation to ignite the shocked flow near the wedge, and forms an oblique detonation in shocked gas to connect with the main oblique detonation.

The oblique detonation wave with the incident $M a 6.6$ is shown in Fig. 6. This is also the Y-shaped shock, but it is more complicated than the structure shown in Fig. 5. In the beginning, the Y-shaped shock moves downstream and the induction zone length reaches its maximum value at the instant shown in Fig. 6. Then the Y-shaped shock will move upstream gradually and the induction length decreases very slowly. When the incident $M a$ decreases further, the oblique detonation structures are similar, but the induction length becomes shorter and shorter, and induces the detached oblique detonation eventually. There is one threshold of induction zone length, below which the boundary layer effect cannot be neglected. However, these effects cannot be studied with the inviscid Euler equations utilized in this paper, which should be addressed in the future work.

Although the fixed Y-shaped shock forms in the case of Ma 6.7, the unstationary process is also involved as shown in Fig. 7. Given uniformed flow field, the Y-shaped shock forms quickly and then moves downstream, as shown in Fig. 7a. The longest induction zone appears at the instant $238.1 \mu$ s, shown in Fig. $7 \mathrm{~b}$. Then the Y-shaped shock moves back to the upstream direction. This process is similar to that in the case of the incident $M a$ 6.6, but the Y-shaped shock stops at the instant $384.0 \mu$ s and then moves downstream again. The Y-shaped shock reaches the final position the instant $533.8 \mu \mathrm{s}$, as shown in Fig. $7 \mathrm{~d}$. The position is about $x=1.53$ and the flow field will not change after a long-time continue simulation. This phenomenon is not studied detailed, although it has been observed before. Han et al. [24] found out unsteady oscillations when they studied the oblique detonation initiation in high temperature gas mixtures. Dong et al. [25] have found out the unstable deflagration-detonation complex appears after the conical shock wave, and its periodic oscillation may have the same mechanism of this unstationary process.

\subsection{Discussion on induction zone structure}

To study the initiation process of the oblique detonation wave, the induction zone length as the function of time is shown in Fig. 8. The induction zone length is defined by the length from the wedge tip to the reaction front near the wedge. With the uniformed flow field, the difference of incident $M a$ is insignificant, so the initial reaction fronts are near $x=1.0$, inducing the length approximately 0.5 for all cases. It can be found out the high $M a$ will induce the quick initiation, but the final induction zone length has a maximum value in the case of the incident $M a$ is 6.7. In the case of Ma 6.8, the initiation is quick and the final length is reached without oscillation. In the case of $M a$ 6.6, the induction zone length rises first, but decreases slowly after the initial stage. Therefore, the Ma 6.7 is the critical Ma corresponding with the maximum induction zone length. To ascertain why the maximum length forms in the case of $M a$ 6.7, the shock/detonation polar curves are plotted in Fig. 9. With the same incident $M a$, the detachment angle of the detonation is smaller than the angle of the shock. Moreover, the detonation detachment angle becomes smaller when the incident $M a$ decreases. It should be noted that the curves are not exact because the heat release and specific heat ratio are not constants in the detailed reaction mechanisms. We deduce that when the incident Ma decreases from 7.0 to 6.0, the detachment angle probably changes from above $30^{\circ}$ to below $30^{\circ}$. The unstationary process is associated with this change, so the critical $\mathrm{Ma}$ 6.7 can be viewed as the smallest $M a$ which can induce detached oblique detonation.

The induction zone length variation demonstrates that there are different mechanisms controlling the induction zone when the incident Ma numbers are different. The induction zone length increases when the Ma decreases from 7.0 to 6.7, which is because the induction zone is dominated by the oblique shock. In these cases, the temperature is low in the induction zone with the small $M a$, so the long ignition delayed time is necessary. However the induction zone length decreases abruptly when the Ma decreases from 6.7 to 6.6 , which is because the induction zone is dominated
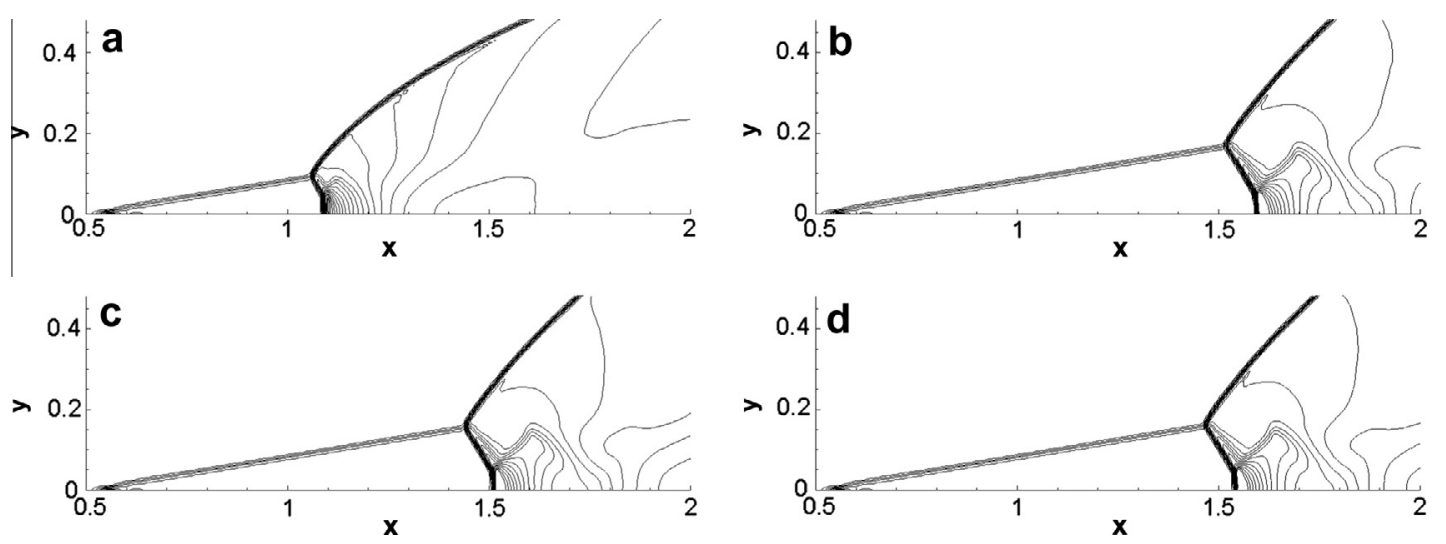

Fig. 7. Pressure of the oblique detonation wave with the incident Ma 6.7 at the instants: $15.4 \mu \mathrm{s}$ (a), $238.1 \mu \mathrm{s}$ (b), $384.0 \mu \mathrm{s}$ (c), $533.8 \mu \mathrm{s}$ (d). 


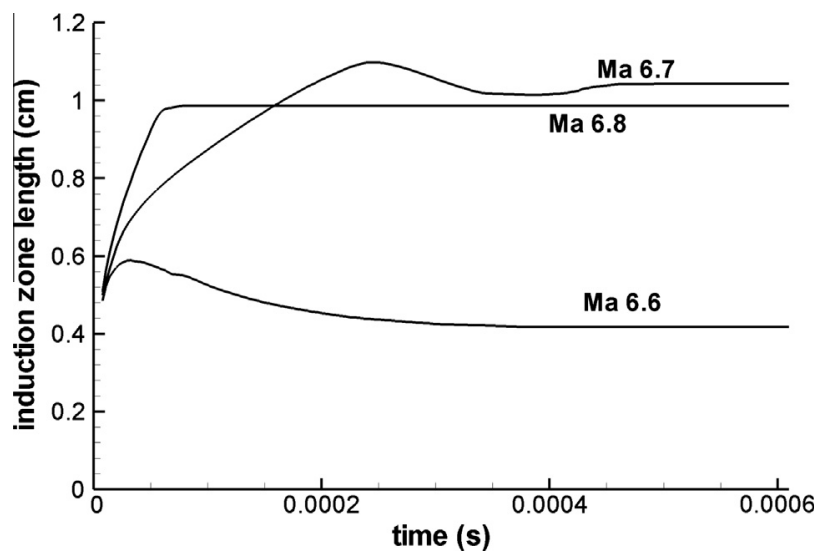

Fig. 8. Induction zone length as function of time in the cases of $M a 6.6,6.7$, and 6.8.

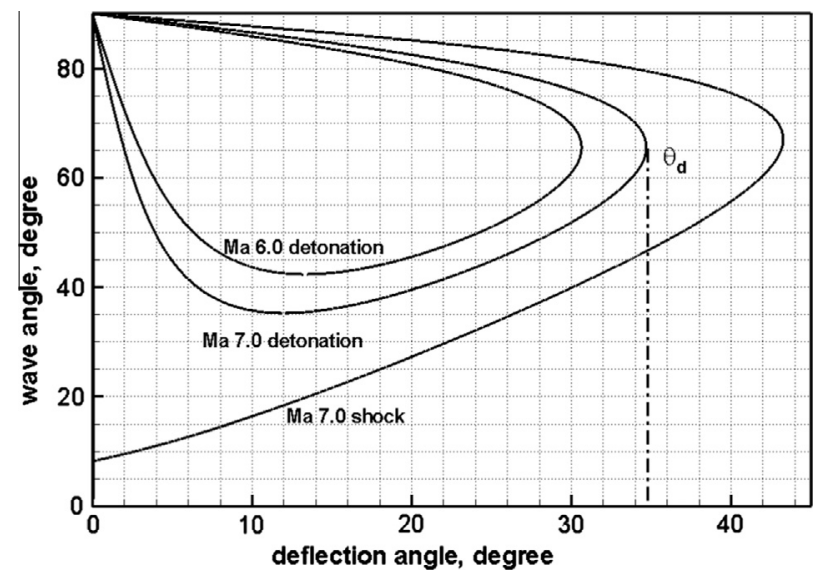

Fig. 9. The shock and detonation polar curves with different incident Ma.

by the oblique detonation wave then. The $\lambda$-shaped shock changes into the Y-shaped shock, so the ignition is achieved by the moderate shock and the long induction length is not necessary. Then the structure is decided by Y-shaped shock in the shocked flow, which is mainly decided by the oblique detonation. Therefore, the dominating factors are different when the incident $M a$ changes across 6.7. It shifts from the oblique shock to the oblique detonation, which induces the maximum induction zone length in the case of Ma 6.7.

\section{Conclusion}

Oblique detonation waves are simulated to study the induction zone structure with different incident $M a$ numbers. Numerical results show three kinds of the shock configurations at the end of the induction zone, which are the $\lambda$-shaped shock, the X-shaped shock and the Y-shaped shock. These three kinds of shock configurations demonstrate the diversity of oblique detonations with low Mach numbers, which cannot be avoided in the oblique detonation propulsion. The X-shaped and Y-shaped shocks appear when the incident $M a$ is low, and the Y-shaped shock associated with the complicated unstationary process. The induction zone length reaches the maximum value when the $X$-shaped shock changes into the Y-shaped shock, in the case of the incident $M a$
6.7, which indicates different mechanisms deciding the induction zone. The oblique shock wave dominates the induction zone when the incident $M a$ is high, while the oblique detonation wave dominates the induction zone when the incident $M a$ is low. Numerical results also suggest that Euler equations are inappropriate in the Y-shaped shock evolution with the low incident Ma. There is one threshold of induction zone length, below which the boundary layer effect cannot be neglected. The threshold study based on viscous simulations should be carried out in the future work.

\section{Acknowledgement}

The research is supported by NSFC Nos. 11372333 and 51376165.

\section{References}

[1] Kailasanath K. Recent developments in the research on pulse detonation engines. AIAA J 2003;41:145-59.

[2] Yuan Li, Tang T. Resolving the shock-induced combustion by an adaptive mesh redistribution method. J Comput Phys 2007;224:587-600.

[3] Ogawa T, Oran ES, Gamezo VN. Numerical study on flame acceleration and DDT in an inclined array of cylinders using an AMR technique. Comput Fluids 2013;85:63-70.

[4] Menes GP, Adelman HG, Cambier JL, Bowles JV. Wave combustors for transatmospheric vehicles. J Propul Power 1992;8:709-13.

[5] Li C, Kailasanath K, Oran ES. Detonation structures behind oblique shocks. Phys. Fluids 1994;4:1600-11.

[6] Viguier C, Figueira da Silva L, Desbordes D, et al. Onset of oblique detonation waves: comparison between experimental and numerical results for hydrogen-air mixtures. Proc Combust Inst 1996;26:3023-31.

[7] Papalexandris MV. A numerical study of wedge-induced detonations. Combust Flame 2000;120:526-38.

[8] Choi JY, Kim DW, Jeung IS, et al. Cell-like structure of unstable oblique detonation wave from high-resolution numerical simulation. Proc Combust Inst 2007; 31:2473-80.

[9] Teng HH, Jiang ZL, Ng HD. Numerical study on unstable surfaces of oblique detonations. J Fluid Mech 2014;744:111-28.

[10] Teng H, Wang C, Zhao W, Jiang Z. Numerical research of the complicated structures on the oblique detonation wave surface. Chin J Theoret Appl Mech 2011;43:641-5.

[11] Gui MY, Fan BC, Dong G. Periodic oscillation and fine structure of wedgeinduced oblique detonation waves. Acta Mech Sin 2011:27:922-8.

[12] Verreault J, Higgins AJ, Stowe RA. Formation of transverse waves in oblique detonations. Proc Combust Inst 2013;34:1913-20.

[13] Figueira da Silva LF, Deshaies B. Stabilization of an oblique detonation wave by a wedge: a parametric numerical study. Combust Flame 2000;121:152-66.

[14] Wang AF, Zhao W, Jiang ZL. The criterion of the existence or inexistence of transverse shock wave at wedge supported oblique detonation wave. Acta Mech Sin 2011;27:311-619.

[15] Teng HH, Jiang ZL. On the transition pattern of the oblique detonation structure. J Fluid Mech 2012;713:659-69.

[16] Teng H, Zhao W, Jiang Z. A novel oblique detonation structure and its stability. Chin Phys Lett 2007;24:1985-8.

[17] Li C, Kailasanath K, Oran ES. Effects of boundary layers on oblique detonation structures. AIAA paper 93-0450. AIAA, Washington, DC; 1993.

[18] Sun M, Takayama K. Conservative smoothing on an adaptive quadrilateral grid. J Comput Phys 1999;150:143-80.

[19] Toro EF. Riemann solvers and numerical methods for fluid dynamics. 2nd ed. Berlin: Springer; 1999.

[20] Kee RJ, Rupley FM, Meeks E, et al. Chemkin-III: a fortran chemical kinetics package for the analysis of gas-phase chemical and plasma kinetics. UC-405, SAND96-8216, Sandia National Laboratories; 1996.

[21] Brown PN, Byrne GD, Hindmarsh AC. VODE, A variable-coefficient ODE solver. SIAM J Sci Stat Comput 1989;10:1038-51.

[22] Sharpe GJ, Quirk JJ. Nonlinear cellular dynamics of the idealized detonation model: regular cells. Combust Theory Model 2008;12:1-12.

[23] Taylor BD, Kasimov AR, Stewart DS. Mode selection in weakly unstable twodimensional detonations. Combust Theory Model 2009;13:973-92.

[24] Han X, Zhou J, Lin ZY. Experimental investigations of detonation initiation by hot jets in supersonic premixed flows. Chin Phys B 2012:21:124702.

[25] Dong G, Fan BC, Li HZ. An investigation on instability of deflagration and detonation induced by conical shock wave. Acta Armamentarii 2010;31: $401-8$ 